Relmecs, diciembre 2018, vol. 8, no. 1, e035, ISSN 1853-7863

Universidad Nacional de La Plata. Facultad de Humanidades y Ciencias de la Educación. Centro Interdisciplinario de Metodología de las Ciencias Sociales.

Red Latinoamericana de Metodología de las Ciencias Sociales

\title{
El trabajo infantil y adolescente del agro argentino: consideraciones sobre su medición en las estadísticas públicas y una propuesta alternativa
}

\author{
Child and young's labor in Argentinean agriculture: considerations \\ on its measurement in public statistics and an alternative proposal
}

\author{
María Marcela Crovetto *, Mercedes Ejarque* y María Virginia Nessi** \\ * CONICET - Instituto de Investigaciones Gino Germani, Facultad de Ciencias Sociales, Universidad de Buenos \\ Aires, Argentina, ** Instituto de Investigaciones Gino Germani, Facultad de Ciencias Sociales, Universidad de \\ Buenos Aires, Argentina | mcrovetto@sociales.uba.ar, mercedes.ejarque@conicet.gov.ar, \\ mvirginianessi@gmail.com
}

\section{PALABRAS CLAVE RESUMEN}

Trabajo infantil y adolescente

Metodología cuantitativa

Mercados de trabajo agropecuarios

KEYWORDS

Children and young labor

Quantitative methodology

Agrarian labor markets
En las últimas décadas, en Argentina, pese al creciente proceso de debate y discusión sobre el trabajo de niños, niñas y adolescentes, es difícil caracterizar el fenómeno a través de estadísticas públicas que permitan mejorar la calidad de las políticas hacia el sector. Este artículo presenta una experiencia alternativa de medición cuantitativa del trabajo infantil y adolescente en distintas áreas no pampeanas de Argentina, particularmente en las actividades vinculadas a los mundos agropecuarios. La indagación y el diseño encuentran sus orígenes tanto en la identificación de las ausencias en tanto objetivos de captación del fenómeno, como en las falencias y las dificultades de relevar estos datos por parte de los instrumentos utilizados para las estadísticas oficiales. Además de una sistematización del estado de la cuestión, en este artículo se exponen los resultados de los relevamientos realizados, que permitieron obtener información específica sobre la ocupación de niños, niñas y adolescentes en trabajo asalariado y como ayuda.

\section{ABSTRACT}

In the previous decades, in Argentina even the increasing process of debate and discussion about children and adolescents labor, it is difficult to characterize the phenomenon through public statistics that could allow improving the public policies quality. This article presents an alternative experience of quantitative measurement in different areas outside the Pampas' region in Argentina, especially those one linked to agriculture. The inquiry and design finds its origin in the identification of either the absences of goals or the failures and difficulties of relieving this information from public statistics. Besides the systematization of the matter, in this article are exposed the survey results, which allow to gather information about children and young occupations, in labor activities and as family help. 


\section{Introducción}

El trabajo infantil en el agro no es un tema nuevo en los estudios sociales agrarios. Sin embargo, la mayoría de las investigaciones realizadas han versado sobre aquello a lo que puede aproximarse desde las estadísticas públicas, los espacios vacíos de relevamiento estadístico oficial, las caracterizaciones cualitativas de la vida de niños, niñas y adolescentes (NNyA) en el contexto de una Argentina agraria de tipo “tradicional” que ya no es la actual.

El inicio temprano en el mundo del trabajo en las actividades agropecuarias ha sido evidenciado históricamente por la investigación social con diferentes problematizaciones. En ese sentido, estudios pioneros en el tema como los realizados por Forni et al. (1979), entre otros, estuvieron enfocados en mostrar qué datos podrían obtenerse de los censos de población y agropecuarios sobre la situación de los niños en los mundos agrarios argentinos, y en señalar los problemas implicados en los estudios sociales sobre el mundo agropecuario por el hecho de no registrar las actividades de los menores de edad. Es muy importante resaltar que hasta entonces se tomaba a la población como económicamente activa desde los 10 años, con lo cual se disponía de más información que en la actualidad cuando la medición se toma a partir de los 14 años.

En investigaciones más recientes se abordan las diversas modalidades de trabajo infantil específicamente. Desde perspectivas de investigaciones cualitativas y con formatos de estudios de caso se encuentran tanto descripciones de procesos de incorporación al mundo del trabajo como también estados de la cuestión sobre el trabajo infantil agrícola en las ciencias sociales (Aguilera y Crovetto, 2009; Aparicio, 2007 y 2009; Aparicio y Crovetto, 2015; Crovetto, 2015; Dahul, Labrunée y Meschini, 2013; Labrunée y Dahul, 2015; Macri et al., 2005; Macri y Uhart, 2012; Miranda Juarez y Re, 2015; Noceti, 2011; Rausky, 2009, Silva, 2010; Re, 2015; Nessi, 2015; Feldman, 1997). Sin capacidad para generalizar sus resultados o evidenciar la problemática a nivel macro -país-, este tipo de trabajos es muy valorado como instancia exploratoria y descriptiva, dado que permite la identificación de dimensiones y variables pasibles de ser sistematizadas luego en estudios de mayor cobertura. Algunos de estos estudios incluso llegan a problematizar sobre las formas de medición del trabajo de NNyA en las estadísticas públicas. Específicamente, Rausky y Santos (2004) reconocían las dificultades de captación de datos sobre este problema a partir de las fuentes existentes, además de las diferencias de criterios utilizados para delimitar y definir el trabajo infantil, las altas tasas de subregistro (en parte por inadecuación de las preguntas a la particularidad temática) y el carácter secundario que se le asigna. Ese trabajo planteaba la necesidad de un relevamiento específico y sistemático. La primera demanda se logró con la Encuesta de Actividades de Niños, Niñas y Adolescentes (EANNA), a fines del mismo año de la publicación, cuyas características y los comentarios sobre la falta de sistematicidad se presentan en el tercer apartado del artículo. En el mismo sentido, y en el marco de un trabajo que presenta el marco conceptual y metodológico de la EANNA, Aizpuru et al. (2005) analizaron los antecedentes nacionales e internacionales para medir el trabajo de NNyA. Algunos de sus aportes son retomados en las siguientes secciones.

A partir de estos antecedentes, este trabajo se propone plantear una síntesis de las ausencias de captación cuantitativa del trabajo de NNyA y presentar una experiencia alternativa de medición del fenómeno que se aplicó en distintas áreas no pampeanas de Argentina. A estos fines, el artículo se ordena en cinco apartados: esta introducción; en segundo lugar, el análisis de las formas de relevamiento del trabajo infantil y adolescente en las estadísticas públicas; luego, las formas e instancias de mediciones específicas sobre el fenómeno desarrolladas desde el Estado con colaboración de organismos internacionales; en cuarto término se presenta el diseño conceptual y metodológico del relevamiento alternativo propuesto y algunos de sus resultados; para finalizar, un epílogo con conclusiones y reflexiones sobre el recorrido realizado.

\section{El relevamiento del trabajo infantil en las estadísticas públicas}

En Argentina existen una serie de estadísticas públicas generales y sectoriales, entre las que se destacan el Censo Nacional de Población, Hogares y Vivienda (CNPHyV), la Encuesta Permanente de Hogares (EPH), la Encuesta Anual de Hogares Urbanos (EAHU), y los censos por rama de actividad, como el agropecuario. Respecto a su 
capacidad de captación del trabajo de NNyA se observan diferentes problemáticas, principalmente en la edad mínima de registro de actividades y en los períodos de referencia tomados por los relevamientos.

En lo referido a la edad mínima de inicio para el registro de la población económicamente activa, las estadísticas públicas han ido elevando el parámetro inferior de la mano de las modificaciones de las leyes laborales y el corrimiento de la edad mínima de admisión al empleo. Ya fue mencionado que el trabajo de Forni et al. (1979) presentaba datos de los mayores de 10 años, pero luego se elevó el registro de las actividades o módulos laborales a personas mayores de 14 años, lo cual coincidía con el límite de edad para la prohibición del trabajo infantil previo a la ley de 2008. La ley 26390 elevó a 16 la edad mínima de admisión al empleo y determinó que entre esa edad y los 18 se permitiera, bajo control y condiciones especiales, el llamado desde entonces trabajo “adolescente" protegido (Nessi, 2015). Esto construye un punto ciego sobre el monitoreo o seguimiento estadístico de la situación laboral de aquellos que están por debajo de esa edad, lo que obstaculiza la intervención y el enfoque de las políticas públicas específicas en un contexto de prohibición explícita del trabajo de menores de 16 años. Si bien la inspección del trabajo ha formado recursos humanos para ello, los mismos no son suficientes, y el procedimiento presenta aún serias dificultades para su implementación plena (puede consultarse detalles en MTESS, 2013). En el mundo agrícola esto revela un problema, pues la incorporación al trabajo (informal o familiar) sucede a edades inferiores, responde a otras historias productivas y familiares y la mayor demanda de mano de obra ocurre en momentos puntuales del año, especialmente en los períodos estivales. Asimismo, está ligado a creencias y representaciones culturales del aprendizaje en el trabajo como juego y como parte de un entrenamiento social (Aparicio, 2009).

En cuanto a los períodos de referencia, en el agro, pero también en otras actividades económicas frecuentes en zonas extrapampeanas (por ejemplo, el turismo) es común la existencia de demandas de mano de obra que varían a lo largo del año. Los relevamientos estadísticos toman la semana anterior como período de referencia para la captación de los ocupados y no resultan adecuados para registrar la ocupación generada por el sector agrario. Así contribuye a subestimar el trabajo transitorio y especialmente aquel de carácter estacional (Aparicio, 2005).

De esta manera, los tiempos de relevamiento y los períodos de referencia utilizados para determinar si se trabaja o no en las estadísticas públicas oficiales no logran captar a la mayoría de los trabajadores estacionales. Por esta razón, y si consideramos que el objetivo de esas mediciones es relevar las características demográficas de la población -especialmente la EPH busca conocer y reflejar más claramente el mercado de trabajo y la situación del empleo-, estas fuentes no son precisas ni siquiera para la recolección de información del trabajo agropecuario estacional de los adultos no registrados, como se expone en Aguilera, Crovetto y Ejarque (2015) $\frac{1}{\text {. }}$

Otra dimensión problemática se constituye por el hecho de que, en que en la Argentina, la ruralidad como concepto que delimita un espacio de residencia y de trabajo lleva años en cuestionamiento en cuanto a su fuerza heurística. La población argentina ha estado abrumadoramente asentada en aglomerados urbanos desde los inicios de su registro en los censos nacionales. Así, se evidenciaron no sólo los crecimientos de los espacios urbanos sino el decrecimiento de la población residente en los rurales (Aparicio y Crovetto, 2010). En el plano conceptual, esto ha generado numerosos debates sobre la importancia empírica y heurística de diferenciar lo rural de lo agrario y lo urbano de lo industrial (Crovetto, 2015). Como el espacio rural se define en el sistema estadístico nacional argentino como toda aglomeración que tenga menos de 2000 habitantes, cerca del $94 \%$ de la población reside en áreas urbanas (según datos del Censo Nacional de Población, Hogares y Viviendas INDEC, 2010). Ello tiene como primera consecuencia la verificación de que los trabajadores agropecuarios -e incluso agroindustrialesresiden en zonas urbanas. Por ello, hablar de la "población rural” como casi equivalente a aquella que realiza trabajo agrario ha dejado de ser una ecuación cierta. También, la estacionalidad en el empleo, que caracteriza al trabajo en el mundo agrícola, tiene como consecuencia que el trabajador de temporada complete su ciclo anual ocupacional en otras ramas de actividad asociadas a labores típicamente urbanas. Aunque incluso en las zonas urbanas podrían detectarse trabajadores agrarios, como los períodos de relevamiento y referencia no coinciden con los ciclos de mayor demanda de trabajo, su captación a través de las fuentes estadísticas es escasa. 
Por último, las características de los mercados de trabajo agropecuario -pago a destajo, escaso nivel de control y cumplimiento de las normativas laborales- y los cambios recientes -creciente urbanización de la mano de obra e incremento de la estacionalidad-impactaron en la ocupación de NNyA en el agro argentino y en sus posibilidades de captación a través de las estadísticas oficiales. En el caso de los relevamientos específicos del agro, especialmente en los censos del sector, al ser respondidos por el productor-empresario, tienden a ocultar a los trabajadores no registrados, lo que incrementa la dificultad de conocer quiénes participan del proceso productivo y cómo lo hacen. Esta situación se complejiza respecto al trabajo familiar ya que se lo tiende a sobredimensionar (en el campo, el productor "siempre trabaja” y la familia también). Sin embargo, es frecuente que se oculte desde qué edad trabajan los niños, las niñas y los adolescentes ya que la prohibición del trabajo hasta ciertas edades (Ley 26.390) y la penalización por violar la edad mínima de admisión al empleo (Artículo 148 bis del Código Penal) son conocidas por los productores.

Superar estas limitaciones (principalmente la edad mínima de registro y el período de referencia) constituye uno de los objetivos de los relevamientos específicos que presentamos en este artículo: conocer cómo y qué formas adopta el empleo en el mundo agropecuario, en qué instancias y cómo participan los miembros del hogar, en especial los niños, las niñas y los adolescentes.

\section{Sobre las mediciones específicas del trabajo de NNyA en Argentina}

En las últimas décadas, la medición del trabajo de NNyA en Argentina ha sido objeto de relevamientos puntuales (algunos desarrollados en el país y otros provenientes del el exterior): el "Módulo para el Monitoreo de Metas Sociales para la Infancia” de la Encuesta Permanente de Hogares (EPH) de mayo de 1994, la Encuesta de Desarrollo Social (EDS) de 1997, la Encuesta de Condiciones de Vida (ECV) de 2001, y el sistema de registro montado por la OIT -el Programa de Información Estadística y de Seguimiento en Materia de Trabajo Infantil (SIMPOC)- desde 1998. Las particularidades de las muestras, formas de relevamiento, y los aciertos y déficits de estos relevamientos se encuentran desarrollados en Aizpuru (2005). Pese a la ausencia de sistematicidad de estos relevamientos y su falta de comparabilidad, estas herramientas y actividades resultaron igualmente una contribución porque se fueron retroalimentando y perfeccionando para acercarse a una caracterización de la situación de los niños y niñas trabajadores (OIT, 2006).

En paralelo con estos relevamientos, el trabajo infantil como problema salió de la esfera exclusiva de estudio académico, como parte de un proceso impulsado principalmente por organismos internacionales como la Organización Internacional del Trabajo (OIT) y el Fondo de las Naciones Unidas para la Infancia (Unicef). Estos organismos ponían en escena los riesgos y los perjuicios que el trabajo realizado por niños y niñas tiene para sus desarrollos integrales. Con un abanico de instrumentos normativos y objetivos de desarrollo a los que Argentina adhiere -pues en 1994 la reforma constitucional incorpora la Declaración Internacional de los Derechos del Niño a la Constitución Nacional- se iniciaba un proceso de debate y construcción de políticas públicas dirigidas a erradicar el trabajo infantil, encontrar las formas de prevenirlo a futuro y proteger el de los adolescentes. El resultado instrumental fue la Ley 26.390 de Prohibición y Erradicación del trabajo infantil y Protección del Trabajo Adolescente, promulgada en 2008, y la creación de organismos del Estado especializados para llevar adelante su cumplimiento. Esto se lograría mediante planes de erradicación y prevención del trabajo infantil, primero a nivel del Estado nacional y luego con la extensión del proyecto hacia los estados provinciales y municipales. De esta manera, se crearon la Comisión Nacional para la Erradicación del Trabajo Infantil (CONAETI), el Observatorio del Trabajo Infantil y Adolescente (OTIA), ambos en el Ministerio de Trabajo, Empleo y Seguridad Social de la Nación (MTESS), y las Comisiones Provinciales para la Erradicación del Trabajo Infantil (COPRETI).

En este marco, en 2012 comenzó un proceso de construcción de mesas locales municipales para la prevención y erradicación del trabajo infantil. Esta iniciativa la encabezó el MTESS -a través de la CONAETI y el OTIA- en articulación con varios ministerios del Poder Ejecutivo Nacional, representantes del sector sindical, la OIT y 
Unicef. Fue una instancia de trabajo con diferentes experiencias de consolidación e institucionalización. Construir información específica fue una meta de ese período porque, desde sus inicios, el problema de la ausencia de datos específicos obstaculizó el diseño de políticas públicas, no sólo a nivel nacional sino además en los niveles locales. Para enfrentar esa ausencia y con el fin de otorgar un mapeo base del problema en el nivel local -que sirviera de insumo para el diseño de políticas de intervención en terreno adecuadas a las realidades de cada municipio y a la modalidad de trabajo infantil allí presente- se llevaron adelante algunos diagnósticos de situación del trabajo infantil y adolescente con enfoque local en casi una decena de municipios, y se realizaron trabajos de investigación en el marco de los formatos de estudios rápidos de la OIT (Crovetto, 2015). Esos estudios buscaron sistematizar los saberes locales dispersos y desnaturalizar situaciones que, por su carácter de históricas, son incorporadas a las prácticas sociales y no son problematizadas. Trabajaron con un perfil cualitativo de tratamiento de la información relevada mediante hojas de vida, entrevistas semiestructuradas y grupos focales compuestos principalmente por familias, autoridades, niños y niñas, adolescentes, docentes, médicos, asistentes sociales, entre otros actores.

Las primeras encuestas y módulos mencionados devinieron en instancias antecesoras para la realización de dos experiencias novedosas en la toma de datos estadísticos sobre este tema en Argentina. Un primer relevamiento oficial con muestreo rural-urbano probabilístico fue la Encuesta de Actividades de Niños, Niñas y Adolescentes (EANNA), realizada entre 2004 y 2006 en algunas regiones de Argentina (AMBA, Mendoza, NOA y NEA). Miembros de este equipo de investigación han participado de las instancias de generación de información previas que dieron sustento al proceso de construcción de conocimiento específico sobre trabajo infantil rural en el marco de la realización de la EANNA. La articulación entre el conocimiento producido en el espacio académico y los organismos nacionales y provinciales de diseño de políticas públicas sobre esta área fue un proceso de retroalimentación. Para ese entonces era un tema que ya ocupaba las indagaciones del equipo y los primeros relevamientos de datos descriptivos comenzaban a hacerse.

La EANNA ha aportado importantes datos para caracterizar el trabajo infantil respecto a qué actividades dentro y fuera del hogar, remuneradas en dinero o de otras formas, realizan los NNyA; los datos incluyen la carga horaria de las mismas. Los resultados de la EANNA en cuanto al trabajo agropecuario se encuentran en Aparicio (2007).

Posteriormente a los relevamientos realizados por este equipo de investigación -cuyos resultados se presentan en el siguiente apartado-, a partir de la EANNA se elaboró el Módulo sobre Actividades de Niños, Niñas y Adolescentes (MANNyA). Éste fue aplicado en el tercer trimestre de 2012 en la Encuesta Anual de Hogares Urbanos (EAHU) realizada por el INDEC (2012). Esta medición y registro lamentablemente sólo se han aplicado en esa oportunidad y tomaron la semana anterior como período de referencia para el relevamiento de actividades de NNyA, lo que subregistra, o no releva directamente, actividades temporarias con picos altos de estacionalidad, como las relacionadas al turismo o las agrarias que tienen lugar en las periferias de los aglomerados relevados, de las cuales suelen participar niños, niñas y adolescentes. Asimismo, se trata de una encuesta en aglomerados grandes e intermedios, por lo cual es difícil captar actividades agrarias estacionales dentro de ese período de referencia, lo que deja por fuera a la mayoría de las actividades productivas que tienen suma importancia en las economías regionales y que dinamizan la vida cotidiana de los aglomerados intermedios del país.

A partir de estos relevamientos, distintos organismos internacionales orientados tanto al trabajo -OIT- como a la infancia -Unicef- han generado informes sobre la situación del trabajo infantil y adolescente en la Argentina (Unicef, s/f; Unicef, 2014; Unicef, 2013). Pero a pesar de los esfuerzos por captar y visibilizar la problemática, no se han generado nuevos relevamientos de datos que le den sistematicidad y mayor cobertura a la medición del trabajo de NNyA. En este marco, la elaboración de una propuesta alternativa para la medición del trabajo infantil y adolescente resultaba necesaria para poder comprender la realidad de los mundos vinculados al agro argentino en las zonas extrapampeanas. 


\section{La experiencia de una propuesta de captación y medición del trabajo de NNyA en diversas regiones de Argentina: diseño y resultados.}

Las experiencias de medición presentadas en este artículo responden a los procesos y dificultades para la caracterización y el análisis de problemáticas vinculadas al trabajo de NNyA a partir de las estadísticas oficiales. Fueron iniciadas como exploraciones cualitativas que buscaban captar las dimensiones que construyen posibles explicaciones y caracterizaciones sobre el trabajo infantil agrícola. Luego, desde 2007, se ha trabajado en el desarrollo y mayor sistematización de un instrumento de medición cuantitativa que fue aplicado en distintas regiones de Argentina y -como se verá- con todos los miembros de los hogares encuestados. Este proceso ha implicado diferentes momentos de discusiones teóricas y metodológicas.

Una primera experiencia fue en ocasión de un trabajo sobre el uso de agroquímicos en las labores de la producción de tabaco en la provincia de Jujuy en el año 2007 (Aparicio, 2009b). Allí se realizaron los primeros relevamientos de datos con preguntas abiertas o semiestructuradas que buscaban conocer las edades de inicio en el trabajo, las tareas que realizaban, con quiénes, para quiénes, y qué tipo de paga recibían. Asimismo, se recogió información sobre otras actividades que podrían no ser consideradas trabajo entre los asalariados del tabaco y que aparecen bajo la forma de "ayudas" o "colaboraciones" con el grupo familiar, ya fuera en un espacio productivo específico como en la reproducción cotidiana del hogar. Este primer relevamiento permitió captar 68 casos, de los cuales 60 son niños y niñas y 8 son adolescentes, pertenecientes todos a familias asalariadas. De ellos, la mayoría desempeñan actividades relacionadas con la ayuda a otros miembros del hogar y sólo una minoría declara trabajar. La participación laboral se incrementa con la edad -es mayor en los adolescentes que en los niños/as- vinculada con la posibilidad de los jóvenes de trabajar por su cuenta, sin intermediación de los padres. Inclusive, al encuestar familias asalariadas del tabaco, se puede observar una transferencia del oficio de adultos a jóvenes. Entre estos NNyA, el porcentaje de mujeres que trabajan es mayor al de las que prestan otros tipos de actividades y se ocupan en labores de encañado, desflore y manejo de estufa, tradicionalmente destinadas a mujeres y niños por su delicadeza y precisión (Aparicio, 2009c). Por último, el relevamiento aportó datos para la caracterización de las actividades laborales: en su mayoría trabajan en jornadas completas a lo largo de por lo menos tres meses (coincidentes con la época de cosecha y de receso escolar: diciembre a marzo). Sin embargo, como el período de referencia toma todos los trabajos realizados en el último año, se pudo relevar que, en ciertos casos, el periodo de trabajo se amplía a ocho meses.

Esta primera recolección de información permitió pensar, pulir y mejorar el proceso de relevamiento de datos para caracterizar al trabajo infantil en el mundo agrícola. La experiencia en Jujuy resultó edificante para la incorporación de una batería de preguntas específicas en el cuestionario a hogares que luego se aplicaría en otras regiones del país.

Con esa información se elaboró el primer cuestionario a hogares, el cual fue sujeto de pruebas piloto (en Tucumán) que permitieron ajustar algunas cuestiones antes del operativo completo. Con la aplicación efectiva del instrumento y el análisis de los resultados en las primeras zonas (Misiones y Chubut, en 2008), se llevó a cabo una revisión completa del formulario con fines de perfeccionarlo para lograr una mejor captación de los temas de investigación, especialmente del trabajo de NNyA. Para ello, se realizó una intensa recopilación de encuestas y censos de la mayoría de los países latinoamericanos y de otros continentes, que ponían en evidencia distintas formas de relevar el trabajo agropecuario. Si bien los contextos y problemas sociológicos podían ser diferentes, en la formulación de cada pregunta y de cada diseño gráfico de cuestionario habían sido puestos en juego recursos, tiempo y conocimientos que resultaban útiles para la mejora del instrumento propio. Esta revisión permitió concretar un nuevo cuestionario (en el cual se aseguró que las modificaciones realizadas no eliminaran la posibilidad de análisis comparativos) que fue aplicado en las últimas dos zonas relevadas (Tucumán - Valle Medio).

De esta manera, la encuesta se aplicó con muestras no probabilísticas en cuatro áreas de Argentina ligadas en sus estructuras sociales y dinámicas socioeconómicas a distintas producciones agropecuarias. En 2008, se realizó en la localidad de Oberá, Misiones, caracterizada por las dinámicas del trabajo en las plantaciones de yerba mate, y 
también en la zona del Valle Inferior del Río Chubut -VIRCh- (Rawson, Trelew, Gaiman y Dolavon), en la provincia de Chubut, ligada a las actividades de la ganadería ovina y la agricultura (hortalizas y forraje). Por último, en 2011, se llevó a cabo en el área citrícola tucumana (las localidades de Tafí Viejo, Cruz Alta, Monteros, Yerba Buena, Burruyacú, Lules y Famaillá), y a su vez en el Valle Medio del Río Negro -VMRN- (en Pomona, Beltrán, Chimpay, Lamarque, Darwin, Belisle y Choele Choel), donde se producen frutas de pepita y hortalizas.

Los temas relevados son similares a los de las encuestas a hogares, aunque también se incluyeron preguntas que permitieron captar las particularidades del trabajo en actividades agropecuarias, como el ciclo ocupacional anual, la intermediación, el pago a destajo y la pluriactividad para todos los integrantes ocupados de un hogar, incluso los NNyA. De operativos que relevan cuestiones vinculadas al empleo se tomaron las preguntas para el rescate de tareas que no suelen ser consideradas trabajo (changas, ayudas en chacras o negocios familiares) e incluso la identificación de trabajos “a los que seguro se va a volver” y que por eso se declara que en ese momento no se está trabajando o que no se busca trabajo -los “disponibles"- (Aguilera, 2007). Estas preguntas permiten captar trabajos estacionales, inclusive en momentos de relevamiento en los que no se esté haciendo dicha actividad. También contribuyen a recoger con mayor precisión el trabajo informal, de mujeres e infantil (cabe mencionar que la forma de captación de la participación femenina en los mercados de trabajo ha sido cuestionada por diversos investigadores desde la década de 1980 (Wainerman, 1997).

Respecto a la captación del trabajo infantil, es importante aclarar que los objetivos de investigación a los que respondió esta serie de encuestas a hogares no propusieron como objeto de estudio especial y particular al trabajo infantil, sino que se propusieron conocer las formas de organización del trabajo en el ciclo ocupacional anual, especialmente en familias asalariadas agrícolas. Así, estos cuestionarios no han incluido los detalles que los formularios de encuesta de las estadísticas oficiales obtuvieron (especialmente los vinculados al uso del tiempo). En dichos relevamientos era importante identificar las formas en las que se organizaba el trabajo en los hogares y capturar, al mismo tiempo, las historias de los adultos respecto de sus inicios en el mundo del trabajo (con preguntas tanto acerca del primer trabajo remunerado, como relacionadas con las actividades que hacían cuando niños, con o sin adultos responsables). Sin embargo, como el cuestionario no establecía límites en cuanto a la definición de la edad (se realizaba toda la batería de preguntas a todos los miembros del hogar) y tampoco en la definición de trabajo (se tomaba la respuesta espontánea de los entrevistados), se podía relevar no sólo el trabajo de NNyA, sino también aspectos culturales, a partir de atender a cuáles eran las tareas que se consideraban como "trabajo".

El cuestionario aplicado fue similar en todas las regiones relevadas, aunque en cada sitio se contó con aliados estratégicos locales (las direcciones provinciales de estadística o investigadores de universidades nacionales) que revisaron los formularios para adaptar el lenguaje a las jergas y regionalismos propios de la zona de relevamiento y producción en cuestión. También contribuyeron en la selección de encuestadores capacitados en metodología o con formación en las temáticas agropecuarias 2 .

Aunque los encuestadores contasen con formación metodológica o teórica, fue fundamental, como en cualquier investigación, la capacitación previa a la salida al campo. Dicha capacitación resalataba la existencia y la importancia del relevamiento de temas de los mercados de trabajo agropecuarios como el trabajo infantil y adolescente, que no son exclusivos pero sí dominantes en esos espacios y que, por lo tanto, resultan de interés para la investigación. Para algunos encuestadores resultaba necesario generar una ruptura con sus prenociones e ideas del sentido común de modo que ellos también pudieran tener una "vigilancia epistemológica” cuando recogieran los datos. Si esto no sucede, los resultados quedan falseados, incompletos y se continúan invisibilizando situaciones particulares del trabajo agrario. Específicamente en lo vinculado al tema de este artículo era pertinente una labor de deconstrucción del prejuicio de los propios encuestadores, quienes, condicionados por la sensibilidad del tema, podían dar por supuesto que los niños y niñas del hogar no trabajaban, por sus edades y condición de infantes. Entonces, aunque se trabajó en la capacitación, luego también se hizo necesario el control del adecuado relevamiento a través de la supervisión in situ. Esto permitió consultar con los encuestadores los casos dudosos 
y/o enviarlos a repreguntar a los hogares cuando había errores en la toma de datos o cuando se presentaban casos en los que los encuestadores no habían realizado la mencionada “vigilancia epistemológica”.

La capacitación también fue necesaria para quienes tuvieron a su cargo las instancias de carga, procesamiento y chequeo de consistencia de las bases de datos; la misma se realizó mediante la explicación de las propias particularidades del lugar relevado y de las decisiones que habían sido tomadas durante el transcurso del trabajo de campo.

Para construir datos en torno al trabajo infantil y adolescente, y lograr una caracterización de mayor profundidad, se utilizaron de la encuesta las siguientes dimensiones de preguntas:

- datos sociodemográficos (edades, sexo, nivel educativo, parentesco),

- ciclo ocupacional anual (actividades realizadas y sus características -época del año de realización, duración, forma de pago, de contrato, de seguridad social, entre otras-),

- historia laboral de entrevistados y familiar (edad del primer trabajo, tipo de actividad realizada, tipo de actividad desarrollada por los padres).

Los relevamientos realizados en las cinco áreas de Argentina permiten obtener algunos datos sobre el trabajo de NNyA que se analizan en el siguiente apartado. La cantidad de casos por zona se presentan en la Tabla 1, en la cual se distingue entre los/as niños/as (menores con hasta 15 años inclusive) y adolescentes (de entre 16 y 18 años, que es la edad permitida por la legislación nacional para el trabajo de menores de edad, para lo cual se requiere el consentimiento de los padres o tutores). Como la encuesta estuvo aplicada a hogares e incluía otros objetivos de investigación, algunas preguntas sobre las características de las ocupaciones de NNyA cuentan con una pequeña base de casos que no permiten un análisis estadístico.

Tabla 1: Distribución de casos según categoría de edad.

\begin{tabular}{|l|l|l|l|l|l|l|}
\hline & \multicolumn{2}{|l|}{ Niños/as } & \multicolumn{2}{l|}{ Adolescentes } & \multicolumn{2}{l|}{ Total de casos } \\
\hline Oberá (2008) & 628 & $90 \%$ & 66 & $10 \%$ & 694 & $100 \%$ \\
\hline VIRCh (2008) & 212 & $84 \%$ & 36 & $16 \%$ & 248 & $100 \%$ \\
\hline Tucumán (2011) & 353 & $90 \%$ & 41 & $10 \%$ & 394 & $100 \%$ \\
\hline VMRN (2011) & 274 & $85 \%$ & 53 & $15 \%$ & 327 & $100 \%$ \\
\hline
\end{tabular}

Fuente: Elaboración propia con base en encuestas a hogares.

\section{Algunos resultados sobre el trabajo de NNyA en distintas áreas de Argentina}

Como ya fue mencionado, las encuestas realizadas relevaron la situación ocupacional de todos los miembros del hogar. Como se observa en la Tabla 2, existen casos de NNyA que estaban trabajando o habían estado haciéndolo durante el último ciclo ocupacional; esto incluye las actividades que realizan dentro y fuera del ámbito doméstico. Frente a la posibilidad de que los encuestados subvaluaran las tareas que realizan, por no considerarlas propiamente “trabajo”, en los relevamientos de Tucumán y Río Negro se utilizaron las mencionadas preguntas de rescate. Los resultados de los relevamientos muestran que, a excepción del VIRCh, en todas las áreas analizadas los entrevistados menores de 18 años han realizado actividades laborales, reconocidas como tales por los propios entrevistados (Tabla 2). 
Tabla 2: Actividades realizadas por niños, niñas y adolescentes según área de relevamiento.

\begin{tabular}{|l|l|l|l|l|}
\hline & Oberá & VIRCh & Tucumán & VMRN \\
\hline Realizan actividades laborales & $5 \%$ & $0 \%$ & $3 \%$ & $4 \%$ \\
\hline Realizan otras actividades & $7 \%$ & $8 \%$ & $4 \%$ & $6 \%$ \\
\hline Sólo concurren a la escuela & $56 \%$ & $69 \%$ & $36 \%$ & $51 \%$ \\
\hline No realizan ningún tipo de actividad & $18 \%$ & $18 \%$ & $50 \%$ & $39 \%$ \\
\hline No sabe/no contesta & $14 \%$ & $5 \%$ & $7 \%$ & $0 \%$ \\
\hline Total & $100 \%$ & $100 \%$ & $100 \%$ & $100 \%$ \\
\hline Cantidad de casos & 694 & 248 & 394 & 327 \\
\hline
\end{tabular}

Fuente: Elaboración propia con base en encuestas a hogares.

Asimismo, se preguntó sobre otros tipos de actividades que realizan los miembros del hogar no reconocidas por ellos mismos como trabajo pero que las pueden igualar en intensidad o cantidad de tiempo dedicado y/o a la contribución que ellas hacen respecto de la reproducción del hogar. Entre éstas se encuentran la ayuda a otros miembros del hogar, las tareas domésticas, las changas y la venta de artesanías y otros productos manufacturados. $\mathrm{Al}$ considerar estas opciones se suman casos de trabajo de NNyA que no son meras ayudas familiares, sino que posibilitan la obtención de ingresos y que resultan invisibilizadas aun por las propias familias.

$\mathrm{Al}$ indagar respecto a los resultados según edad, se pueden caracterizar las particularidades de cada grupo etario. Si bien, la categoría Niño/a engloba a todos aquellos individuos hasta 15 años inclusive, se optó por diferenciar en dos subcategorías: hasta 13 años, y de 14 a 15 años. Esta distinción permitirá visibilizar aquellos casos que no son relevados por las estadísticas oficiales anteriormente descritas. En el caso de los niños y las niñas (menores de 15 años, inclusive), en todas las regiones se encuentran casos que trabajan o realizan changas, actividades domésticas o ayudas para obtener ingresos (Tabla 3), lo que evidencia la presencia de trabajo infantil. De esta manera, resulta elocuente que, de haberse sostenido la misma forma de medición que la de las estadísticas oficiales, esto no habría sido registrado. Las actividades de "ayuda" tienen mayor incidencia entre los menores de 14 años. Al pasar a la categoría de niños y niñas de 14 y 15 años empieza a igualarse la cantidad de casos que realizan ambas actividades, a excepción de Chubut que aún no presenta registros en las actividades laborales.

Tabla 3: Actividades realizadas por niños, niñas por grupos de edad, según área de relevamiento.

\begin{tabular}{|c|c|c|c|c|c|c|c|c|}
\hline \multirow{3}{*}{ Categoría de edad } & \multicolumn{8}{|c|}{ Niños y Niñas } \\
\hline & \multicolumn{4}{|c|}{ Hasta 13 años } & \multicolumn{4}{|c|}{ De 14 a 15 años } \\
\hline & Oberá & VIRCh & Tucumán & VMRN & Oberá & VIRCh & Tucumán & VMRN \\
\hline $\begin{array}{l}\text { Realizan actividades } \\
\text { laborales }\end{array}$ & $1 \%$ & $0 \%$ & $0 \%$ & $1 \%$ & $14 \%$ & $0 \%$ & $6 \%$ & $4 \%$ \\
\hline $\begin{array}{l}\text { Realizan changas, } \\
\text { ayudas o actividades } \\
\text { domésticas }\end{array}$ & $5 \%$ & $6 \%$ & $3 \%$ & $6 \%$ & $14 \%$ & $6 \%$ & $4 \%$ & $6 \%$ \\
\hline $\begin{array}{l}\text { Sólo concurren a la } \\
\text { escuela }\end{array}$ & $57 \%$ & $63 \%$ & $32 \%$ & $43 \%$ & $58 \%$ & $94 \%$ & $61 \%$ & $79 \%$ \\
\hline $\begin{array}{l}\text { No realizan ningún } \\
\text { tipo de actividad }\end{array}$ & $23 \%$ & $26 \%$ & $57 \%$ & $49 \%$ & $3 \%$ & $0 \%$ & $22 \%$ & $11 \%$ \\
\hline No sabe/no contesta & $14 \%$ & $5 \%$ & $7 \%$ & $0 \%$ & $10 \%$ & $0 \%$ & $8 \%$ & $0 \%$ \\
\hline Total & $100 \%$ & $100 \%$ & $100 \%$ & $100 \%$ & $100 \%$ & $100 \%$ & $100 \%$ & $100 \%$ \\
\hline Cantidad de casos & 559 & 176 & 302 & 227 & 69 & 36 & 51 & 47 \\
\hline
\end{tabular}


Fuente: Elaboración propia con base en encuestas a hogares.

Estos resultados se vinculan a la percepción negativa que se tiene sobre el trabajo de niños y niñas, pero conforme aumenta la edad igualmente empieza a enunciarse. También, porque al ser cada vez mayores se independizan de sus padres, y trabajan directamente para el empleador (Re, 2014; Aparicio y Crovetto, 2015; Roa, 2012; Nessi, 2015).

Entre los adolescentes (16-17 años), aunque las bases de análisis son menores, el trabajo tiene mayor presencia (Tabla 4). En las provincias norteñas el número de los adolescentes que trabajan incluso iguala o supera al de aquellos que sólo concurren a la escuela o al de los que no realizan ninguna actividad.

Tabla 4: Actividades realizadas por adolescentes según área de relevamiento.

\begin{tabular}{|l|l|l|l|l|}
\hline & \multicolumn{4}{l|}{ Adolescentes (16-17 años) } \\
\hline & Oberá (2008) & VIRCh (2008) & Tucumán (2011) & VMRN (2011) \\
\hline Realizan actividades laborales & $32 \%$ & $0 \%$ & $22 \%$ & $13 \%$ \\
\hline Realizan otras actividades & $15 \%$ & $19 \%$ & $7 \%$ & $8 \%$ \\
\hline Sólo concurren a la escuela & $42 \%$ & $72 \%$ & $32 \%$ & $60 \%$ \\
\hline No realizan ningún tipo de actividad & $2 \%$ & $0 \%$ & $37 \%$ & $19 \%$ \\
\hline No sabe/no contesta & $9 \%$ & $8 \%$ & $2 \%$ & $0 \%$ \\
\hline Total & $100 \%$ & $100 \%$ & $100 \%$ & $100 \%$ \\
\hline Cantidad de casos & 66 & 36 & 41 & 53 \\
\hline
\end{tabular}

Fuente: Elaboración propia con base en encuestas a hogares.

Si bien las diferencias por sexo son mínimas, existe un predominio del sexo masculino en las actividades laborales de todas las regiones. Según Roa (2012) y Vazquez Laba (2007), la explicación radica en que los hombres son los primeros en ingresar, individualmente o como ayuda a otros miembros, a los mercados de trabajo. Sin embargo, estos estudios también muestran cómo empieza a insertarse el trabajo femenino en actividades agropecuarias, tanto por sus calificaciones tácitas como por el crecimiento de familias monoparentales con mujeres como cabeza de familia (Vazquez Laba, 2009).

\section{Caracterización de ocupaciones realizadas por NNyA}

Los relevamientos permitirían caracterizar las actividades laborales que realizan los NNyA, especificando las formas en que son contratados, trasladados a los lugares de trabajo, cantidad de horas y meses ocupados, otras actividades realizadas y percepción de seguridad social. Sin embargo, debido a que la cantidad de casos es pequeña en la mayoría de las áreas, no se presentan los datos numéricos sino las tendencias observadas.

En Misiones, la mitad de los NNyA que trabajan se dedican a la realización de actividades vinculadas con la producción agropecuaria: primordialmente en la yerba mate, pero también en la cosecha del té. No es un dato menor si se considera que en la región de análisis el trabajo adulto por excelencia es el destinado a la yerba, lo que indica que el traspaso generacional de ocupaciones se encuentra presente. En segundo lugar, se encuentran actividades relacionadas a la reproducción del hogar, como ser el cuidado de niños, el empleo doméstico o la jardinería. Estas tareas luego conllevan a desarrollar un vínculo laboral en otros hogares.

Entre los varones, habría mayor incidencia de la actividad agropecuaria (yerba y té) y de servicios y comercio, que entre las mujeres. Sólo ellas realizan trabajo doméstico y cuidado de niños. Estas diferencias responden a una 
división sexual del trabajo y al predominio de la jefatura masculina: hay un posterior ingreso de los hombres a trabajos no relacionados con el hogar, mientras que las mujeres quedan muchas veces centradas en el ámbito doméstico y sólo algunas trabajando en la yerba o en otras actividades laborales, como el comercio y los servicios. Esta cuestión ya ha sido analizada en otros mercados de trabajo agropecuarios en el mundo adulto (Vázquez Laba, 2007; Aparicio, 2012).

Respecto a la edad, a diferencia de lo que sucedía con la ayuda familiar y las tareas domésticas, tanto niños como adolescentes se ocupan en las mismas actividades laborales. Sólo cabe mencionar que en el paso a la adolescencia se reduce la diversidad de actividades realizadas por los jóvenes.

En Tucumán, se presentan dos actividades que realizan los NNyA: agropecuarias y en la construcción. La edad es el atributo con el cual se hace observable la diversificación de actividades: mientras que los niños solo se dedican a tareas vinculadas a la cosecha, en la adolescencia empiezan a desempeñarse en la construcción. Esta última fortalece una inserción autónoma de los adolescentes en el mundo del trabajo. A su vez, en la construcción solo se ocupan varones, mientras que las mujeres no participan. A diferencia de otras regiones analizadas, las mujeres no se insertan en el mercado vinculadas al hogar de terceros, sino que realizan dichas actividades en el ámbito propio, como amas de casa.

Las actividades realizadas en el Valle Medio del Río Negro se vinculan, en primer lugar, a diversas cosechas (tomate, frutas); en segundo lugar, a trabajos en aserraderos y servicios (mozos, servicio de catering, fletes, por ejemplo). La edad está vinculada con la diversificación de actividades, ya que los niños se ocupan en la producción de materias primas (madera y agricultura) y cuando entran a la adolescencia suman su participación en otras ramas de la economía. La diversidad de ocupaciones se encuentra solo en los hombres, ya que las mujeres declararon trabajar en tareas agrarias.

De los NNyA trabajadores en todas las regiones, sólo un mínimo porcentaje realizaría una segunda ocupación. Son casos que se dan en la región de Misiones y VMRN, y suele ser también dentro de la rama agropecuaria.

En todas las regiones, los NNyA tienen jornadas de trabajo de más de 7 horas, una magnitud mayor que la cantidad de horas permitida por ley para trabajadores adolescentes. Asimismo, en su mayoría se ocupan durante todo el año o solo algunos meses coincidentes con el período escolar. Esta situación no les permitiría la realización de otras actividades, especialmente la asistencia a la escuela.

Respecto al pago de aportes de seguridad social o adicionales establecidos por ley, en la región de Tucumán, ninguno de los trabajadores adolescentes (mayores de 16 años que pueden estar registrados con permiso de sus padres) los recibiría. Pero en Valle Medio y Misiones, algunos declaran que reciben obra social, aguinaldo, aportes jubilatorios y proporcional por vacaciones.

\section{Consideraciones finales}

Las experiencias de relevamiento en las distintas zonas de Argentina muestran que en todas ellas existen NNyA que trabajan o realizan actividades que pueden estar incluidas en las definiciones de trabajo infantil estricto o ampliado como está expuesto en la Ley 26.390. Al respecto, la extensión del rango de edad con relación al tomado por las estadísticas oficiales permitió la captación de diferencias en las actividades realizadas según la edad de los NNyA, lo que demostró que existe, no solo una diversificación durante el ciclo ocupacional anual, sino también en los rangos etarios (los menores combinan actividades laborales y domésticas, mientras que a medida que crecen se concentran en el trabajo). Sobre las características de las actividades realizadas, los NNyA mostrarían similitudes con las de los adultos: división sexual de tareas y precariedad en las formas de inserción laboral.

En las regiones analizadas, en algunos casos combinan estas ocupaciones con la asistencia a la escuela, pero en general las jornadas o los momentos del año en que realizan las tareas dificultarían la escolaridad de quienes trabajan. Solo en Chubut no hay casos registrados de NNyA que únicamente trabajen. De esta manera, se estaría 
presentando una tensión entre las posibilidades de asistencia a la escuela y las de trabajar. Sin embargo, cabe tener en cuenta que los relevamientos se realizaron antes o fueron coincidentes con el proceso de implementación de la Asignación Universal por Hijo -AUH- (2009), lo cual tiene dos consecuencias. Por un lado, que la tensión escuela-trabajo en los NNyA, que históricamente fue una dimensión de análisis en el trabajo infantil, y que solía ser utilizada para explicar el abandono escolar, puede en la actualidad haber sufrido modificaciones debido a la aplicación de la AUH. Este aspecto no estaba presente en el momento del relevamiento en Chubut y Misiones (2008) y sí lo estaba cuando se hicieron los operativos de Tucumán y Valle Medio del Río Negro. Entonces, la segunda consecuencia es que las diferencias regionales observadas pueden responder tanto a características socioculturales y productivas como a los impactos de esta política pública de protección de la infancia. Esto sucede tanto respecto a la tensión escuela-trabajo como en otro fuerte argumento, mencionado en los debates explicativos sobre la presencia del trabajo infantil, que es la experiencia formativa en el trabajo de los jóvenes.

De esta manera, las investigaciones cualitativas y de casos permiten profundizar en las causas y los motivos de los procesos y fenómenos que se observaron en estos relevamientos que, por las características de las muestras y la cantidad de casos registrados, no permiten aplicar procedimientos estadísticos de carácter explicativos que permitan construir tendencias generalizables.

El recorrido realizado por la historia de los relevamientos oficiales muestra que han habido algunos intentos por mejorar la forma de captación y medición del trabajo infantil. Sin embargo, el principal problema para que estas mediciones contribuyan al conocimiento de la problemática y al mejoramiento de las políticas públicas para la erradicación del trabajo infantil es su sistematicidad. Asimismo, el límite de edad para el registro de la población económicamente activa es un obstáculo que persiste en los censos y otras estadísticas oficiales que son sistemáticas. En lo relativo específicamente al trabajo de NNyA en tareas agropecuarias también se sostiene la semana anterior como fecha de referencia que, como en el caso de los adultos, no permite captar las actividades estacionales, lo que genera un subregistro de la contribución de estos trabajos al desarrollo del sector. Como muestran los datos de los relevamientos realizados y otras investigaciones, la utilización de instrumentos de medición adaptados a la problemática y al sector agrario permite evidenciar la presencia de trabajo de NNyA en diversos lugares de Argentina. Por ello, una mejora en las estadísticas oficiales, que incluso considere los aportes y experiencias realizados en el ámbito público -como la EANNA-, puede contribuir no sólo a captar mejor esta situación sino a obtener información para mejorar las políticas públicas destinadas a erradicar el trabajo infantil y garantizar el desarrollo integral de los niños, las niñas y los adolescentes.

\section{Agradecimientos}

Este proceso de investigación fue financiado a través de diferentes proyectos de Ciencia y Técnica de organismos públicos nacionales. Agradecemos los comentarios de los evaluadores anónimos de este trabajo.

\section{Notas}

1 Estas observaciones no están basadas en cuestionamientos acerca de la muestra del relevamiento en el caso de la EPH, sino de su diseño conceptual. Como ya se mencionó en su diseño no se pregunta acerca del trabajo de menores y las ocupaciones temporarias, a través de lo cual se podrían captar muchos trabajadores del agro que residen en ciudades grandes e intermedias, como dan cuenta muchas investigaciones recientes (Aparicio y Benencia, 2014; Craviotti, 2008; Neiman y Bardomás, 2001; Riella y Mascheroni, 2015, entre otros)

2 Más detalles de las características del relevamiento pueden encontrarse en Autor, 2015. 


\section{Referencias Bibliográficas}

Aizpuru, A., Bisconti, F., Debowicz, D., Herger, N., López, L., Lorenzetti, A. (...) y Viú, G. (2005). Medición y estimaciones de trabajo infantil en el contexto de la encuesta de actividades de niños, niñas y adolescentes. $7^{\circ}$ Congreso Nacional de Estudios del Trabajo. Recuperado de: www.aset.org.ar/congresos/7/17006.pdf

Aguilera, M. E. (2007). ¿Se van para volver? Trabajadores migrantes y mercado de trabajo en el Alto Valle del Río Negro 1995-2005 (Tesis de maestría en Demografía Social, inédita). Universidad Nacional de Luján, Luján, Argentina.

Aguilera, M. E. y Crovetto, M. M. (2009). El trabajo infantil rural en dos valles de la Patagonia argentina. En Trabajo infantil. Investigación y acción. Sistematización de los trabajos presentados en los Seminarios Regionales. 2008 - 2009, 1-14. Buenos Aires: Ministerio de Trabajo, Empleo y Seguridad Social, CONAETI, UNICEF, PNUD, OIT.

Aparicio, S. (2005). Trabajo y trabajadores en el sector agropecuario de la Argentina. En N. Giarracca y M. Teubal (coords.), El campo en la encrucijada (pp. 193-221). Buenos Aires: Alianza Editorial.

Aparicio, S. (2007). El trabajo infantil en el agro. En El trabajo infantil en la Argentina. Análisis y desafíos para la política pública [205-240]. Buenos Aires: Ministerio de Trabajo, Empleo y Seguridad Social de la Nación y Organización Internacional del Trabajo.

Aparicio, S. (2009). Niños trabajadores en el agro argentino. Familias campesinas y de asalariados rurales. Mitos y creencias en torno al trabajo infantil rural. XXVII Congreso de la Asociación Latinoamericana de Sociología. Asociación Latinoamericana de Sociología. Buenos Aires.

Aparicio, S. (2009b). Tabaco, Mercado de Trabajo y Cultura en Jujuy. Buenos Aires: Superintendencia de Riesgos del Trabajo, Ministerio de Trabajo y Seguridad Social de la Nación. Recuperado de: http://biblioteca.srt.gob.ar/Publicaciones/2009/Tabaco Jujuy.pdf

Aparicio, S. (2009c, junio). Trabajos y relaciones de trabajo en la producción tabacalera empresarial. XXVIII Congreso de la Asociación de Estudios Latinoamericanos. Río de Janeiro, Brasil.

Aparicio, S. (2012). Algunos antecedentes sobre el trabajo femenino en la agricultura argentina. En Empleo y condiciones de trabajo de mujeres temporeras agrícolas [11-81]. Roma Italia: FAO.

Aparicio, S. y Crovetto, M. (2010, abril) Un objeto de estudio complejo: los mercados de trabajo "rururbanos". VI Congreso de la Asociación Latinoamericana de Sociología del Trabajo. México D.F., México.

Aparicio, S., y Crovetto, M. (2015, enero- junio). Los jóvenes en el agro argentino: inicios tempranos en el mundo del trabajo. Carta Económica Regional, 27(115), 90-113.

Benencia, R. y Aparicio, S. (2014). Nuevas formas de contratación en el trabajo agrario. Buenos Aires: Ciccus.

Craviotti, C. (2008) Empleo agrario y ruralidad ampliada. Geograficando, 4(4). 99 -116. Recuperado de: http://www.fuentesmemoria. fahce.unlp.edu.ar/art_revistas/pr.3741/pr.3741.pdf

Crovetto, M. (2015). La investigación del trabajo infantil desde un enfoque local. La participación en proyectos diagnósticos, la dimensión metodológica y el análisis de resultados. En Prevención y erradicación del trabajo infantil: diagnóstico, evolución reciente y desarrollo de experiencias locales, capítulo 5 [205-231]. Buenos Aires: OTIA - $\quad$ MTESS - $\quad$ OIT - $\quad$ Unicef. Recuperado de: http://www.trabajo.gob.ar/left/estadisticas/otia/centroDoc/verDocumento.asp?id=232

Dahul, M. L., Labrunée, M. y Meschini, P. (2013). Trabajo Infantil: Una mirada desde la percepción de las familias de los sectores pobres urbanos de la zona puerto-sur de la ciudad de Mar del Plata. INFEIES - RM, 2(2), 33-67 Recuperado de: http://www.infeies.com.ar 
Feldman, S., García Mendez, E. y Araldsen, H. (1997). Los niños que trabajan. Buenos Aires, UNICEF, $14,942$.

Forni, F., Aparicio, S., Asano, S., Benencia, R., Novick, M., Orsatti, Á., Tort, M. I. y Vasilachis, I. (1979). Un primer diagnóstico sobre el trabajo infantil en la República Argentina. Documento de Trabajo N6. Buenos Aires: CEIL.

INDEC (2012). Módulo sobre actividades de niños, niñas y adolescentes (MANNyA). Informe técnico. Diseño de registro y estructura de la base de microdatos. Módulo aplicado durante el tercer trimestre de 2012. Buenos Aires.

Labrunée, M. E. y Dahul, M. L. (2015, agosto) Protección social para el abordaje de la problemática del trabajo infantil en el cordón frutihortícola del partido de General Pueyrredón. Las miradas e intervenciones posibles por parte de las instituciones educativas en el marco de la institucionalidad vigente. $12^{\circ}$ Congreso Nacional de Estudios del Trabajo. El trabajo en su laberinto. Viejos y Nuevos Desafíos. Buenos Aires.

Macri, M., Ford, M., Berliner, C. y Molteni, M. J. (2005). El trabajo infantil no es un juego. Estudios e investigaciones sobre trabajo infanto-adolescente en Argentina (1990-2003). Buenos Aires: Editorial Stella, La Crujía.

Macri, M. y Uhart, C. (2012). Trabajos infantiles e infancias. Investigaciones en territorio (Argentina, 20052010). Buenos Aires: La Crujía Ediciones.

MTESS (2013). La Inspección del Trabajo en la Argentina. 2003-2012. Acciones y Resultados. Buenos Aires: Secretaría de Trabajo, Ministerio de Trabajo, Empleo y Seguridad Social de la Nación.

Miranda Juarez, S., y Re, D. A. (2015). El trabajo infantil rural en México y la Argentina. El caso de dos complejos agroindustriales. Revista Sociedad Y Economía, 91-106.

Neiman, G. y Bardomás, S. (2001). Continuidad y cambio en la ocupación agropecuaria y rural de la Argentina. En G. Neiman (comp.), Trabajo de campo. Producción, tecnología y empleo en el medio rural (pp. 11-30) Buenos Aires: Ediciones CICCUS.

Nessi, M. V. (2015, Octubre). Trabajo infantil, estrategias familiares y migraciones en los mercados de trabajo agropecuarios en regiones no pampeanas. Un estado de la cuestión. II Jornadas de Jóvenes Investigadores UNSAM- IDAES. Buenos Aires.

Noceti, M. B. (2011). "Trabajo infantil rural" y "explotación laboral infantil rural": Aportes antropológicos a la diferenciación de conceptos para el diseño de políticas de protección de derechos del niño en el sudoeste bonaerense. Papeles de trabajo, (22), 58-73. Recuperado de: http://www.scielo.org.ar/scielo.php? script=sci_arttext\&pid=S1852-45082011000200005\&lng=es\&tlng=es.

OIT (2006). Infancia y Adolescencia: trabajo y otras actividades económicas. Primera encuesta. Análisis de resultados en cuatro subregiones de la Argentina. Buenos Aires: Miño y Dávila Editores.

Rausky, M. E. (2000). Perspectivas sobre el trabajo infantil en la Argentina: Un análisis de las investigaciones desarrolladas en el campo de las Ciencias Sociales. Revista de estudios regionales y mercado de trabajo, 5, 177200.

Rausky, M. E. y Santos, J. (2004). El relevamiento del trabajo infantil en las encuestas a hogares de Argentina: un primer acercamiento para el caso de la provincia de Bs As y sus principales aglomerados urbanos. VI Jornadas de Sociología. Facultad de Ciencias Sociales, Universidad de Buenos Aires, Buenos Aires. Recuperado de: http://www.aacademica.org/000-045/309

Re, D. A. (2014). La producción de tabaco Virginia en la provincia de Jujuy. Movilidad social y productores agrarios (Tesis de doctorado en Ciencias Sociales, inédita). Universidad de Buenos Aires, Buenos Aires, Argentina. 
Re, D. A. (2015). La “ayuda” infantil en la tarefa de yerba mate. Cultura, mercado y legislación. Conflicto Social, 8(14), 221-242.

Riella, A. y Mascheroni, P. (2015). Asalariados rurales en América Latina. Montevideo: CLACSO.

Roa, M. L. (2012). Los jóvenes de familias tareferas de los barrios periurbanos: ¿la generación del cambio? Psicoperspectivas. Individuo Y Sociedad, 11(1), 108-133.

Silva, M. A. (2010). Trabajo infantil rural en Argentina. GeoTrópico, 7, 1-14. Recuperado de: http://www.geotropico.org/

Vazquez Laba, V. (2007). Desorganizando la tradicional división sexual del trabajo familiar: un estudio comparativo de familias asalariadas rurales del Noroeste Argentino (Tesis de Doctorado en Ciencias Sociales, inédita). Universidad de Buenos Aires, Buenos Aires, Argentina.

Vazquez Laba, V. (2009). Hacia una complejización de la tipología de familias. Los modelos familiares en el Noroeste Argentino. Revista de Ciencias Sociales, IV-I (126-127), 105-113.

Wainerman, C. (1997). La invisibilidad censal de las mujeres trabajadoras. En C. Wainerman y R. Sautu (comps.), La trastienda de la investigación [187-221]. Buenos Aires: Editorial de Belgrano. 